\title{
REMOCIÓN DE ÁCIDO SULFHÍDRICO POR MICROORGANISMOS SOBRE LODOS ACTIVADOS EN AGUAS RESIDUALES DE LA INDUSTRIA ALIMENTICIA
}

\author{
REMOVAL OF HYDROGEN SULFIDE BY MICROORGANISMS ON ACTIVATED \\ SLUDGES IN THE WASTEWATER OF FOOD INDUSTRY
}

\author{
Luis Miguel Santillán Quiroga, Lorena Paola Paredes Pacheco
}

Escuela Superior Politécnica del Chimborazo ESPOCH, Código Postal: EC060155. Dirección: Panamericana Sur km 1 1/2, Riobamba-Ecuador

*Autor para correspondencia: luis.santillanquiroga@espoch.edu.ec

\begin{abstract}
Resumen
La importancia del siguiente trabajo radica en implementar un tratamiento de remoción de $\mathrm{H}_{2} \mathrm{~S}$ con un bajo impacto ambiental. El objetivo fue el de evaluar microorganismos eficientes para la remoción de ácido sulfhídrico sobre lodos activados de las aguas residuales industriales. Se construyó un biofiltro con microorganismos eficientes, lodo activado y material filtrante. Se inocularon durante tres semanas en lodos 21 litros de microorganismos eficientes activado, controlando parámetros como $\mathrm{pH}$, temperatura, humedad, oxígeno y luz solar, garantizando las condiciones aptas de desarrollo de bacterias fotosintéticas, ácido lácticas y levaduras. El muestreo del agua residual se realizó en la caja de revisión más cercana al punto de descarga al alcantarillado de La Ibérica, y para su análisis a la salida del biofiltro, tomando una muestra con tres repeticiones cada semana, durante 21 días, para conocer la concentración inicial, intermedia y final de $\mathrm{H}_{2} \mathrm{~S}$ y el porcentaje de remoción alcanzado en el tratamiento. Los resultados de la investigación dictaminaron que los microorganismos eficientes aumentaron la carga microbiana de los microorganismos endógenos en el lodo, consiguiendo un mejor crecimiento de la biopelícula y logrando la reducción de la concentración de $\mathrm{H}_{2} \mathrm{~S}$ de 3,68 mg/L a 0,45 mg/L, alcanzando un porcentaje de remoción del 70,35\%. El presente trabajo analiza que a mayores tiempos de retención se da una mejor actuación de microorganismos eficientes en el tratamiento. La utilización de microorganismos eficientes en la remoción de $\mathrm{H}_{2} \mathrm{~S}$, resulta rentable por sus tiempos cortos de actuación y bajos costos de aplicación.
\end{abstract}

Palabras claves: remoción, ácido sulfhídrico, microorganismos eficientes, muestreo, lodo activado, biopelícula. 


\begin{abstract}
The importance of this work is the implementation of a natural treatment with low environmental impact. The objective was to evaluate effective microorganisms for the removal of hydrogen sulfide on activated sludges of the industrial wastewaters. For the experimental part of the research a biofilter was built with effective microorganisms, activated sludge and filter material. 21 liters of activated EM were inoculated on sludges for over 3 weeks, controlling parameters such us $\mathrm{pH}$, temperature, humidity, oxygen and sunlight, guaranteeing the optimal conditions for the develop of photosynthetic bacteria, lactic acid and yeast. Sampling of wastewater was carried out in the closest manhole to the point of discharge of the sewage system of La Iberica and for its analysis at the biofilter output, taking a sampling with three repetitions each week, for 21 days, to know the initial, intermediate and final concentration of $\mathrm{H}_{2} \mathrm{~S}$ and the removal percentage reached during the treatment. Research findings showed that the effective microorganisms increased the microbial load of the endogenous microorganisms in the sludge, obtaining a better growth of the biofilm and achieving the reduction of the $\mathrm{H}_{2} \mathrm{~S}$ concentration from $3.68 \mathrm{mg} / \mathrm{L}$ to $0.45 \mathrm{mg} / \mathrm{L}$, reaching a removal percentage of $70.35 \%$. This work analyses that to a greater retention times, a better performance of the effective microorganisms is given. The use of effective microorganisms in the removal of $\mathrm{H}_{2} \mathrm{~S}$ is profitable since the time of action is short and the costs of application are low.

Keywords: Removal, Hydrogen sulfide, efficient microorganism, sampling, activated sludge, biofilm.

Forma sugerida de citar: Santillán Quiroga, L. M. y Paredes Pacheco, L. P. 2018. Remoción de ácido sulfhídrico por microorganismos sobre lodos activados en aguas residuales de la industria alimenticia. La Granja: Revista de Ciencias de la Vida. Vol. 27(1):112-123. http: //doi.org/10.17163/lgr.n27.2018.09.
\end{abstract}




\section{Introducción}

El ácido sulfhídrico se genera a partir de una reacción biológica en los sistemas de desagüe o plantas de tratamiento de aguas residuales, siendo el causante de la corrosión de los sistemas de alcantarillado (Bas, 2017). Las aguas residuales con rangos de $\mathrm{pH}$ por debajo de 5.0 generan un impacto ambiental, ya que al ser descargadas directamente a los ríos provocan malos olores y la pérdida de su biota nativa (Fernández-Alba et al., 2006).

La mayor parte de la generación del $\mathrm{H}_{2} \mathrm{~S}$ ocurre en la capa de lodo que se forma en las paredes de la tubería o en los depósitos que se forman en la base de ésta. Si el agua servida contiene poco o nada de oxígeno disuelto, la difusión del $\mathrm{H}_{2} \mathrm{~S}$ se produce debido a las condiciones anaeróbicas existentes (Arriagada Monreal, 2008).

Actualmente en el Ecuador los procesos utilizados para la remoción de ácido sulfhídrico en las aguas residuales envuelven tratamientos químicos en gran medida como la utilización de coagulantes, productos clorados, la fotocatálisis con luz, un semiconductor $\left(\mathrm{TiO}_{2}\right)$ como catalizador y un agente oxidante como el peróxido de hidrogeno $\left(\mathrm{H}_{2} \mathrm{O}_{2}\right)$ que involucran costos elevados y menor accesibilidad hacia todos los sectores (Varela Reyes, 2013). Sin embargo, hoy en día, existen otros métodos biotecnológicos que involucran la utilización de microorganismos para su tratamiento biológico, buscando aumentar la capacidad de implementar procesos más amigables con el ambiente (Szymanski y Patterson, 2003).

La importancia de esta investigación radica en utilizar microorganismos eficientes como una alternativa accesible frente al problema de la contaminación en el área del tratamiento biológico de las aguas residuales, no solo por involucrar precios económicos sino también por sus tiempos cortos de remoción.

La Tecnología de Microorganismos Eficientes ${ }^{\mathrm{TM}}$ (EM ${ }^{\mathrm{TM}}$ por sus siglas en inglés) es un tratamiento natural de menor impacto desarrollado por el Prof. Teruo Higa en la Universidad de Ryukyus, Okinawa, en el sur de Japón, a partir de 1982 (EEAITAJ, 2013). Originalmente, fue utilizado como alternativa para los fertilizantes químicos y pesticidas, sin embargo, el uso de la Tecnología EM ${ }^{\mathrm{TM}}$, en las dos últimas décadas, se ha expandido de la agricultura al tratamiento de aguas y efluentes, control de malos olores e innumerables tratamientos industriales
(Madigan et al., 2004).

El presente trabajo aspira ser un documento con aporte práctico y teórico para las futuras investigaciones en la evaluación y aplicación de microorganismos eficientes para la reducción de ácido sulfhídrico y demás compuestos contaminantes de las aguas residuales industriales en nuestro país.

Al desarrollarse el presente trabajo investigativo los beneficiarios directos serán principalmente las industrias, al obtener un sistema de tratamiento biológico de menor costo que les permita reducir un mayor porcentaje de ácido sulfhídrico antes de su descarga, en menor tiempo y cumpliendo la norma ambiental vigente.

\section{Materiales y métodos}

\subsection{Técnicas e instrumentos de tabulación de datos}

El análisis estadístico se realizó en el programa SPSS, mediante pruebas no paramétricas de Kolmogorov-Smirnov de una muestra y ANOVA de un factor, permitiendo analizar e interpretar los datos cuantitativos. Según la Norma de Calidad Ambiental y de Descarga de Efluentes: Recurso Agua, Libro VI Anexo 1, el límite máximo permisible de $\mathrm{H}_{2} \mathrm{~S}$ para aguas dulces, frías o cálidas, y en aguas marinas y de estuario es $0,0002 \mathrm{mg} / \mathrm{l}$.

Para la cuantificación de $\mathrm{H}_{2} \mathrm{~S}$ se utilizó el método de azul de metileno (Standard Methods 4500-S2$\mathrm{D} / \mathrm{HACH}$ 8131), se basa en la reacción de sulfuros de hidrógeno y sulfuros metálicos solubles en ácido con N, N-dimetil-p-fenilendiamina sulfato para producir azul de metileno. La intensidad del color azul es proporcional a la concentración de sulfuro presente en la muestra. La lectura se realiza en un espectrofotómetro a $664 \mathrm{~nm}$.

\subsection{Etapas de la investigación}

\subsubsection{Primera etapa: Proceso de activación e inocu- lación de microorganismos eficientes}

Los microorganismos del producto EM-1, está compuesto por bacterias fotosintéticas, bacterias ácido lácticas y levaduras, que se encuentran en estado de latencia, para la activación se siguió la "Guía Técnica de la Tecnología EM" de EMRO (EMPROTEC, 2016) siendo AGEARTH su representante oficial en Ecuador. 
A los 7 días de su fermentación los microorganismos antes mencionados están activados y listo para utilizarse siempre y cuando presenten un $\mathrm{pH}$ por debajo de 4.00 un olor agridulce agradable y un cambio de coloración de café oscuro a café anaranjado (EMPROTEC, 2016).

La caracterización microbiológica del lodo utilizado como sustrato, se realizó antes y después de incorporar los microorganismos eficientes a la unidad de análisis, para describir la concentración tanto de patógenos: mohos y levaduras, coliformes fecales y totales como de microorganismos mesófilos aerobios presentes (Galvis Toro y Rivera Guerrero, 2013). Para la evaluación de estos parámetros microbiológicos se utilizó la misma metodología establecida en la norma, variando únicamente en los medios de cultivo, las placas utilizadas, tipo de siembra y temperatura de incubación (INEN, 2013).

Durante las tres semanas de tratamiento se inoculó 1 litro de Microorganismos Eficientes activado cada 6 horas en el lodo y se revolvió cada semana para permitir su aireación.

\subsubsection{Segunda etapa: Muestreo del agua residual an- tes del tratamiento}

La toma del agua residual se llevó a cabo en la caja de revisión más cercana al punto de descarga al alcantarillado de la industria procesadora de alimentos y su traslado fue inmediato para su posterior refrigeración y almacenamiento.

Para los análisis iniciales de laboratorio, se tomaron 3 muestras, cada una con 3 repeticiones, de un total de 15 muestras recolectadas, antes de hacer pasar el agua residual por la unidad de análisis para conocer la concentración inicial de $\mathrm{H}_{2} \mathrm{~S}$ presente en las aguas a tratarse (Coronel Pazmiño, 2015).

El proceso de muestreo se realizó en tres fases, en la primera fase, se recolectó de 15 muestras una muestra de 3 litros, dándonos un total de 45 litros, en la segunda fase se obtuvieron 15 litros que fueron recogidos por 5 días, en la tercera fase, al igual que la segunda, se recogieron 15 litros, en 5 días de muestreo, dándonos un total de 75 litros de agua residual industrial para ser utilizada en la investigación.

\subsubsection{Tercera etapa: Construcción de la unidad de análisis (Biofiltro)}

La unidad se construyó en base a las dimensiones establecidas de un biofiltro a escala de laboratorio diseñado por (Higuera, Arroyave y Flores, 2009) para reducir el índice de contaminación por cromo generado en las industrias del curtido de cueros. Con esta referencia, se procedió a la construcción de un biofiltro con las siguientes dimensiones, como se muestra en la Figura 1: altura $37.2 \mathrm{~cm}$, largo $32 \mathrm{~cm}$ $\mathrm{y}$ ancho $31.3 \mathrm{~cm}$.

El material utilizado fue vidrio transparente de $8 \mathrm{~mm}$ de espesor con un orificio de $8 \mathrm{~cm}$ de diámetro en el centro de su base para la salida del agua. Se colocó además una malla para evitar el paso de sólidos, una llave de paso para controlar el flujo de agua, cuatro mangueras con orificios colocadas en cada esquina del biofiltro para la aireación de los lodos y una manguera con agujeros simétricos para que el agua se distribuya hacia toda la unidad de análisis que contiene lodos activados, EM (Microorganismos Eficientes) y un material filtrante. 


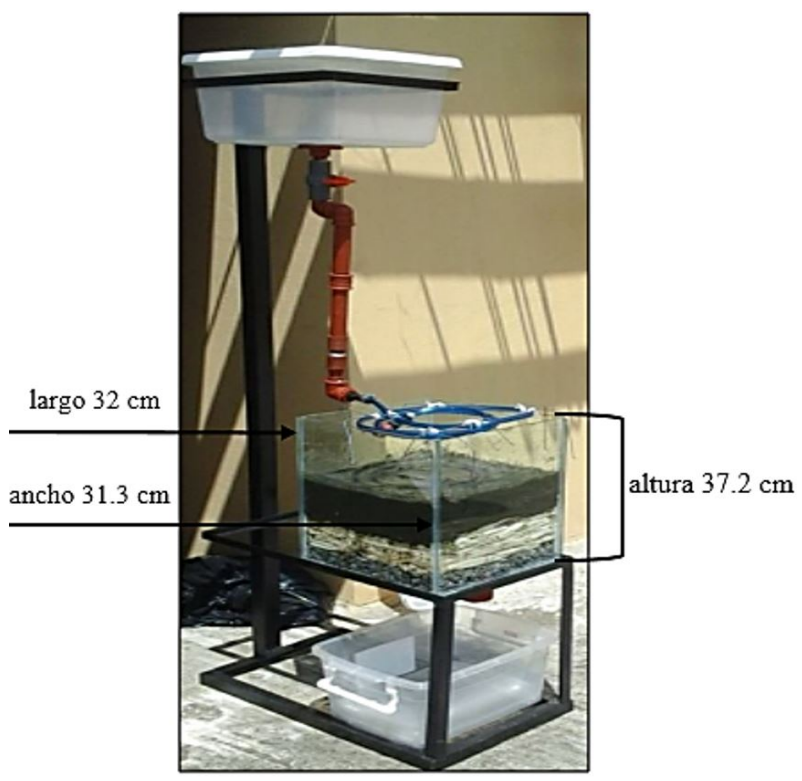

Figura 1. Biofiltro a escala de Laboratorio.

\subsubsection{Cuarta etapa: Establecimiento de las condicio- nes aptas para los microorganismos eficientes en el Biofiltro}

Una vez construida la unidad de análisis se procedió a monitorear las variables de control aptas dentro del biofiltro para el desarrollo y multiplicación de los microorganismos eficientes, considerándose como los más importantes $\mathrm{pH}$, temperatura, humedad.

\section{Control de $\mathrm{pH}$}

Los microorganismos eficientes contienen bacterias fotosintéticas, ácido lácticas y levaduras que necesitan de un $\mathrm{pH}$ ácido para su correcto desarrollo y multiplicación (Moreno y Velarde, 2016). Por lo tanto, se realizó un control antes y durante el tratamiento para asegurar que el $\mathrm{pH}$ del lodo se mantenga entre un rango de acidez constante para asegurar la supervivencia de los microorganismos dentro del biofiltro.

La lectura del $\mathrm{pH}$ se tomó con la ayuda de tiras de papel indicadoras de $\mathrm{pH}$. Este parámetro se monitoreo sumergiendo la tira sobre una muestra acuosa fresca de lodo (cada semana) y se comparó el color obtenido con la escala contenida en el empaque para comparar su grado de acidez o alcalinidad (UTE, 2014).

\section{Control de temperatura}

El monitoreo de la temperatura se realizó diariamente con la ayuda de un termómetro digital, teniendo en cuenta que a temperaturas altas los microorganismos actúan con mayor eficiencia, por lo que este parámetro debe oscilar de $30-45{ }^{\circ} \mathrm{C}$ durante todo el tratamiento (Lázaro, 2014). Para mantener una estabilidad adecuada dentro de la unidad de análisis, y considerando que el clima del lugar es variable, se montó un invernadero, que ayudó a controlar dicho parámetro (Galvis Toro y Rivera Guerrero, 2013).

\section{Control de humedad}

Este parámetro es importante ya que el lodo debe mantenerse siempre húmedo dentro del biofiltro, ya que si la humedad baja, los microorganismos no se desarrollan por no tener el agua suficiente para su metabolismo, por lo que se considera que la humedad debe estar entre 50 - 70\% (Salinas Carrillo y Huacollo Álvarez, 2011).

Para determinar la humedad se consideró realizar el método empírico de la prueba de puño (Novoa et al., 2008). La técnica nos dice que si el sustrato apretado no toma la forma del puño cerrado y no gotea, la humedad aproximada es menor al $70 \%$, por el contrario si al agarrar una cantidad del sustrato que alcanza con el puño de una mano se escu- 
rren más de 10 gotas en 1 minuto, significa que la humedad está en un $80 \%$ aproximadamente.

Teniendo en cuenta la técnica antes detallada se tomó con la mano una muestra de lodo del centro del biofiltro y se apretó fuertemente el mismo, al salir un hilo de agua continuo, se consideró que la muestra se encuentra entre un rango correcto de humedad ( $>70 \%$ aproximadamente).

\subsubsection{Quinta etapa: Muestreo del agua tratada}

Durante el tratamiento con microorganismos eficientes, se tomaron tres muestras de agua filtrada, cada una con 3 repeticiones. El muestreo se realizó en frascos de plástico de 1 litro, rotulados de la siguiente manera: M1 (EM), para la muestra 1 del tratamiento con microorganismos eficientes; M2 (EM), muestra 2 del tratamiento con microorganismos eficientes y M3 (EM), muestra 3 del tratamiento con microorganismos eficientes (Coronel Pazmiño, 2015).

El primer muestreo se realizó en la semana uno de tratamiento, se tomó la muestra de agua filtrada, con 3 repeticiones para conocer la concentración de ácido sulfhídrico y se realizaron monitoreos adicionales de $\mathrm{pH}$, conductividad y turbidez, para evaluar la incidencia de dichos parámetros en la reducción de $\mathrm{H}_{2} \mathrm{~S}$. El segundo y tercer muestreo se realizó cada 11 días, ya que en este tiempo los microorganismos se estabilizan (Jusoh, Manaf y Latiff, 2013).

\section{Resultados y discusión}

\subsection{Caracterización microbiológica del lo- do activado}

Como se muestra en la Figura 2, se realizó un análisis antes de inocular los microrganismos eficientes en el lodo (día 1) que permitió identificar la presencia de mesófilos aerobios, coliformes totales, coliformes fecales, mohos y levaduras, de la misma manera se realizó un análisis después de los 15 días de haber empezado con el tratamiento, con el fin de evaluar el comportamiento que tuvieron los EM (Microorganismos Eficientes) ante los cambios de las variables de control. El resultado se muestra en la Tabla 1.

Tabla 1. Parámetros microbiológicos evaluados en el lodo activado.

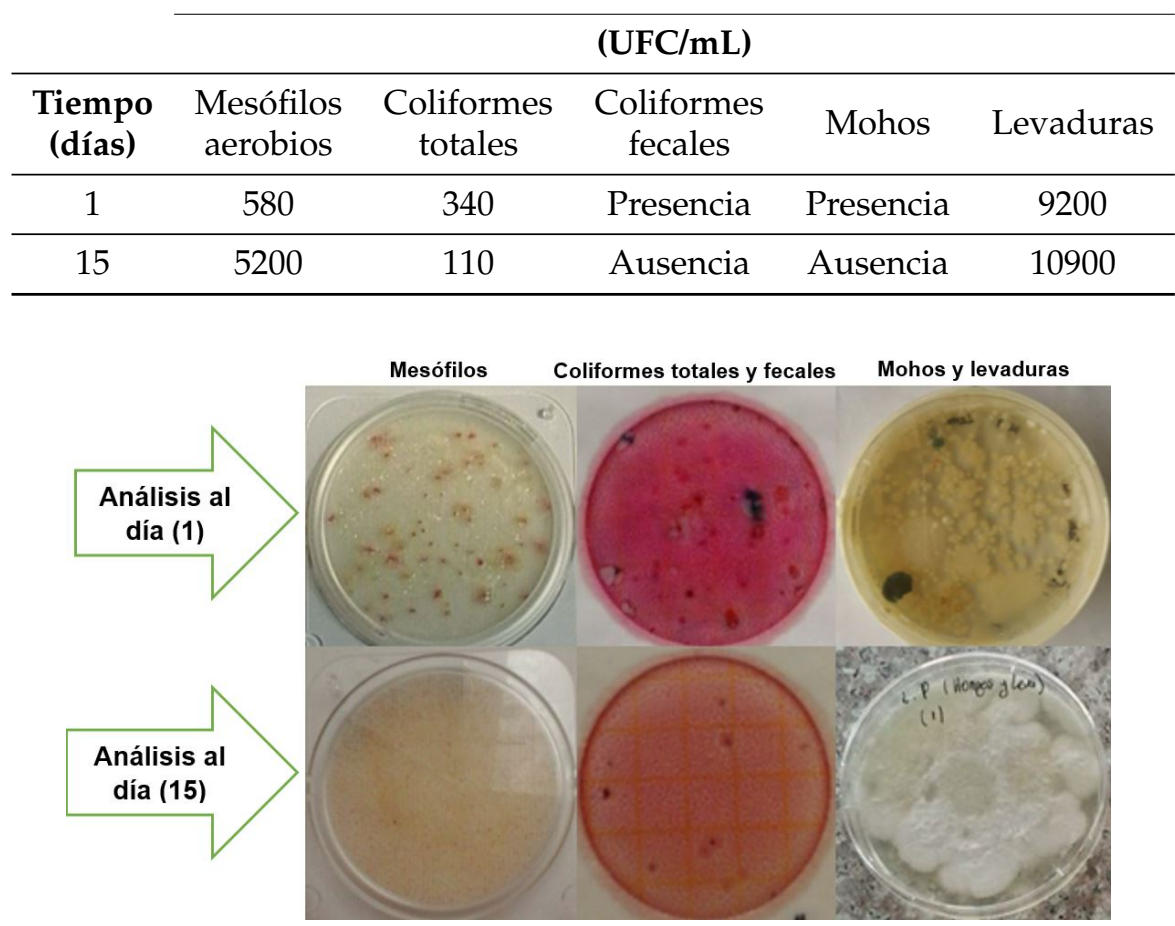

Figura 2. Crecimiento en placa de microorganismos. 


\subsection{Control de condiciones aptas para la activación y desarrollo de los microor- ganismos eficientes en el lodo activado.}

La Figura 3 recoge los resultados del comportamiento de $\mathrm{pH}$ y temperatura durante la activación y el período de 21 días en los que se llevó a cabo el control para su adaptación. En cuanto a la activación, se cumple una relación inversamente proporcional, ya que mientras más días pasan para su fermentación el $\mathrm{pH}$ disminuye. A partir del cuarto día la fermentación se acelera mostrando una disminución de $\mathrm{pH}$ significativa de 4,55 mientras que al séptimo día alcanza un valor de 3,33. Por lo tanto el producto de Microorganismos Eficientes al día 7 cumple con un rango de acidez menor a 4,00 considerado como óptimo para su activación y apto para su utilización (Organic Fruits, 2016).
La temperatura a los primeros días indica variaciones de 28 a $30^{\circ} \mathrm{C}$, mientras que al pasar el tiempo se visualizan variaciones de 32 a $35^{\circ} \mathrm{C}$, como se visualiza en la Figura 3. La línea de tendencia muestra que la temperatura aumenta y disminuye a un ritmo constante. Por lo tanto, a pesar de las variaciones ambientales externas, se observa una tendencia a mantener condiciones estables de temperatura cálida de alrededor de $30-35^{\circ} \mathrm{C}$ durante el tratamiento.

En cuanto al $\mathrm{pH}$ tiende a mantener un rango de acidez constante, comprendida entre un valor de 45 , con una tendencia lineal durante los 21 días de tratamiento, lo que hace del sustrato un medio adecuado para el crecimiento de microorganismos fotosintéticos, acido lácticos y levaduras (García et al., 2008).

\section{Comportamiento de $\mathrm{T}^{\circ}$ y pH monitoreados diariamente}

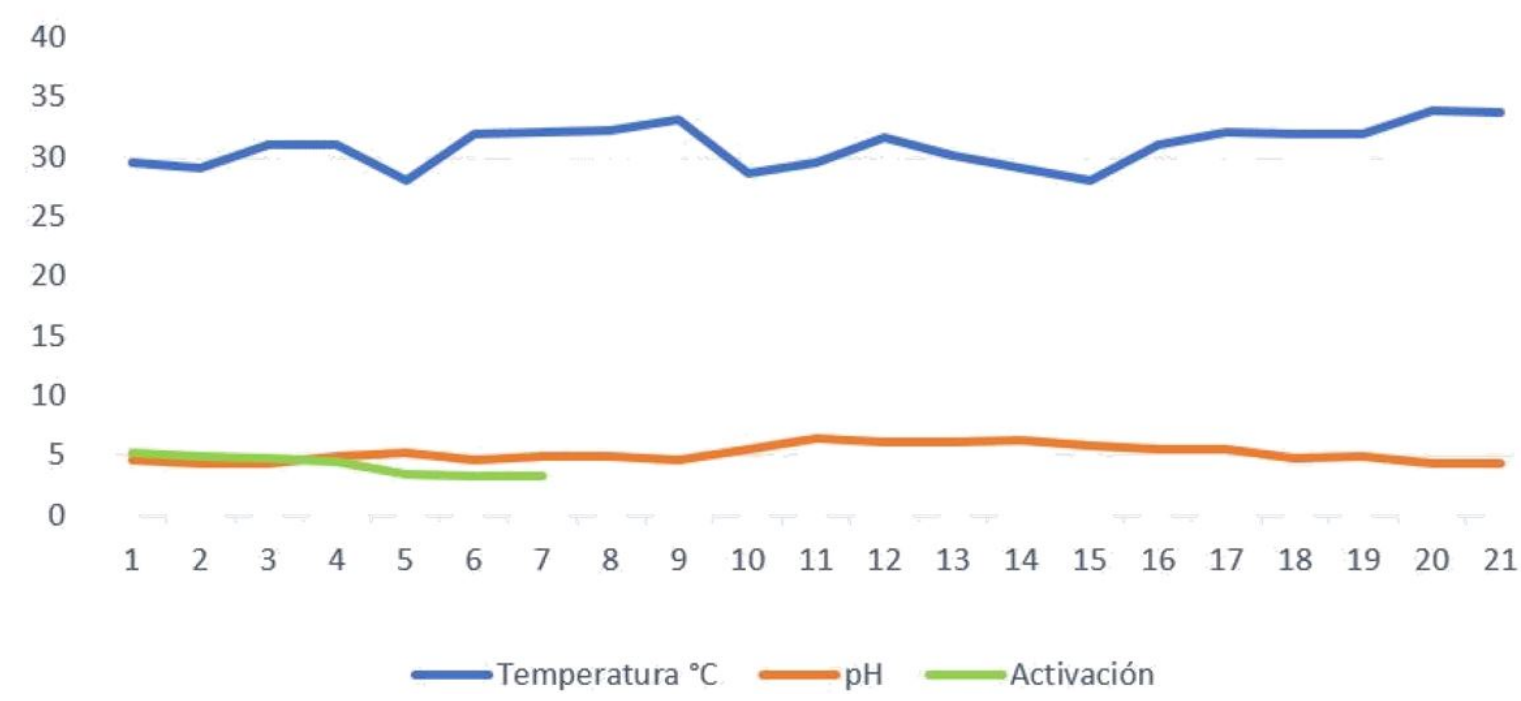

Figura 3. Comportamiento de $\mathrm{T}^{\circ}$ y $\mathrm{pH}$ monitoreados diariamente.

\section{Análisis de la concentración de ácido sulfhídrico}

Con el fin de comprobar si el tratamiento con microorganismos eficientes (factor), durante las tres se- manas, tuvo resultados favorables en la remoción de ácido sulfhídrico de las aguas residuales se realizó un diseño completamente aleatorio en el programa SPSS (Tabla 2). 
Tabla 2. Análisis de Anova de un factor.

\begin{tabular}{|c|c|c|c|c|c|c|}
\hline \multicolumn{7}{|c|}{ ANOVA de un factor } \\
\hline & & $\begin{array}{l}\text { Suma de } \\
\text { cuadrados }\end{array}$ & $\mathrm{gl}$ & $\begin{array}{l}\text { Media cua- } \\
\text { drática }\end{array}$ & $\mathrm{F}$ & Sig. \\
\hline \multirow{3}{*}{$\mathrm{H} 2 \mathrm{~S}$} & Inter-grupos & 11,416 & 2 & 5,708 & 24,566 & 0 \\
\hline & Intra-grupos & 1,394 & 6 & 0,232 & & \\
\hline & Total & 12,81 & 8 & & & \\
\hline \multirow{3}{*}{$\mathrm{pH}$} & Inter-grupos & 6,883 & 2 & 3,442 & 6883,4 & 0,000 \\
\hline & Intra-grupos & 0,003 & 6 & 0,001 & & \\
\hline & Total & 6,886 & 8 & & & \\
\hline \multirow{3}{*}{ Conductividad } & Inter-grupos & 16,692 & 2 & 8,346 & 23473,63 & 0,000 \\
\hline & Intra-grupos & 0,002 & 6 & 0,000 & & \\
\hline & Total & 16,694 & 8 & & & \\
\hline \multirow{3}{*}{ Turbidez } & Inter-grupos & 18716,67 & 2 & 9358,333 & 1238,603 & 0,000 \\
\hline & Intra-grupos & 45,333 & 6 & 7,556 & & \\
\hline & Total & 18762 & 8 & & & \\
\hline
\end{tabular}

En este caso, Anova de un factor está determinado por el valor de significancia, que es aproximadamente 0 en el análisis de las variables $\mathrm{H}_{2} \mathrm{~S}$ $(\mathrm{p}=0.001), \mathrm{pH}(\mathrm{p}=0.000)$, conductividad $(0.000)$ y turbidez ( $\mathrm{p}=0.000)$; por lo tanto, al tener un resultado de $\mathrm{p}<0.05$, se rechaza la hipótesis nula y se acepta la hipótesis alternativa, lo que muestra que existen diferencias entre las semanas de tratamiento, con al menos un par de comparaciones de todas las posibles. Esto quiere decir que las semanas influyen sobre la concentración de ácido sulfhídrico, $\mathrm{pH}$, conductividad y turbidez en las aguas residuales a tratarse.

Tabla 3. Análisis descriptivo de la variable $\mathrm{H}_{2} \mathrm{~S}$ por semanas de tratamiento con EM

\section{Descriptivos}

$\mathrm{H} 2 \mathrm{~S}$

Intervalo de confianza

N Media Desviación típica Error típico para la media al 95\% Mínimo Máximo

Límite Límite

inferior superior

\begin{tabular}{ccccccccc}
\hline 1 & 3 & 3,170 & 0,815 & 0,470 & 1,145 & 5,194 & 2,237 & 3,687 \\
\hline 2 & 3 & 1,426 & 0,150 & 0,086 & 1,052 & 1,800 & 1,274 & 1,570 \\
\hline 3 & 3 & 0,446 & 0,100 & 0,058 & 0,196 & 0,696 & 0,346 & 0,541 \\
\hline Total & 9 & 1,681 & 1,265 & 0,421 & 0,708 & 2,653 & 0,345 & 3,688 \\
\hline
\end{tabular}


En la Tabla 3, se muestra las medias del $\mathrm{H}_{2} \mathrm{~S}$ en cada semana de tratamiento con microorganismos eficientes, en la que se observa que tienen diferencias de una semana en relación a la otra, siendo la media de la semana 3 la que presenta un valor menor en comparación de la semana uno y dos. En cuanto a la desviación típica se presentan similares en la semana 2 y 3, a excepción de la semana uno en donde se observa una desviación típica menor. El intervalo de confianza del $95 \%$ muestra estimaciones por intervalos de la ubicación de la verdadera media poblacional del $\mathrm{H}_{2} \mathrm{~S}$ en relación con cada una de las semanas de tratamiento, con los valores mínimos y máximos del tiempo en semanas que se llevó a cabo el tratamiento.

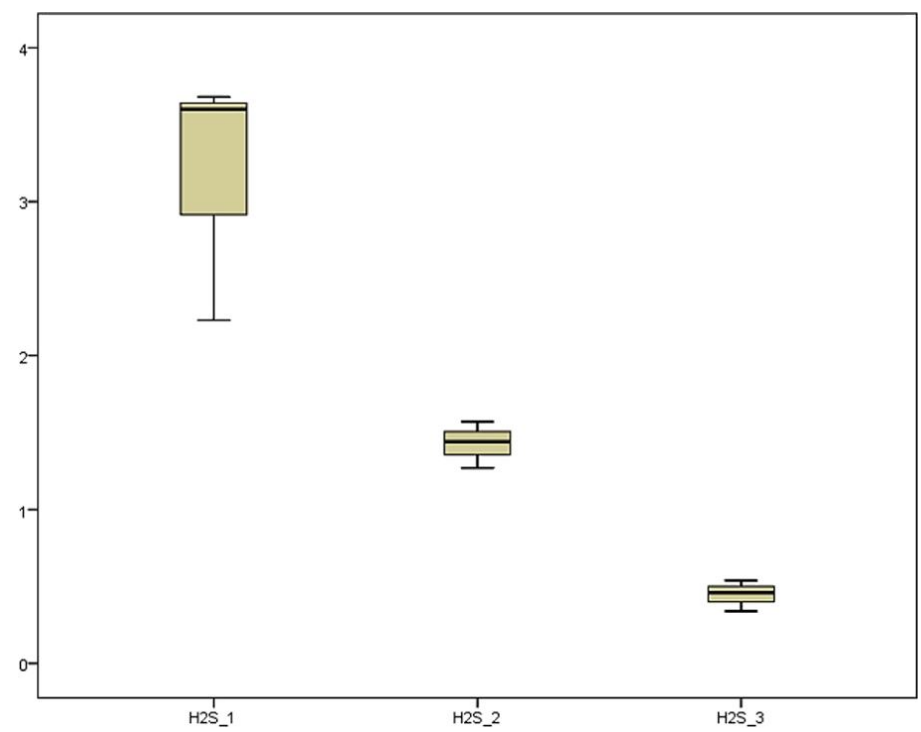

Figura 4. Comparativo de la concentración $\mathrm{H}_{2} \mathrm{~S}$ durante tres semanas de tratamiento con Microorganismos Eficientes.

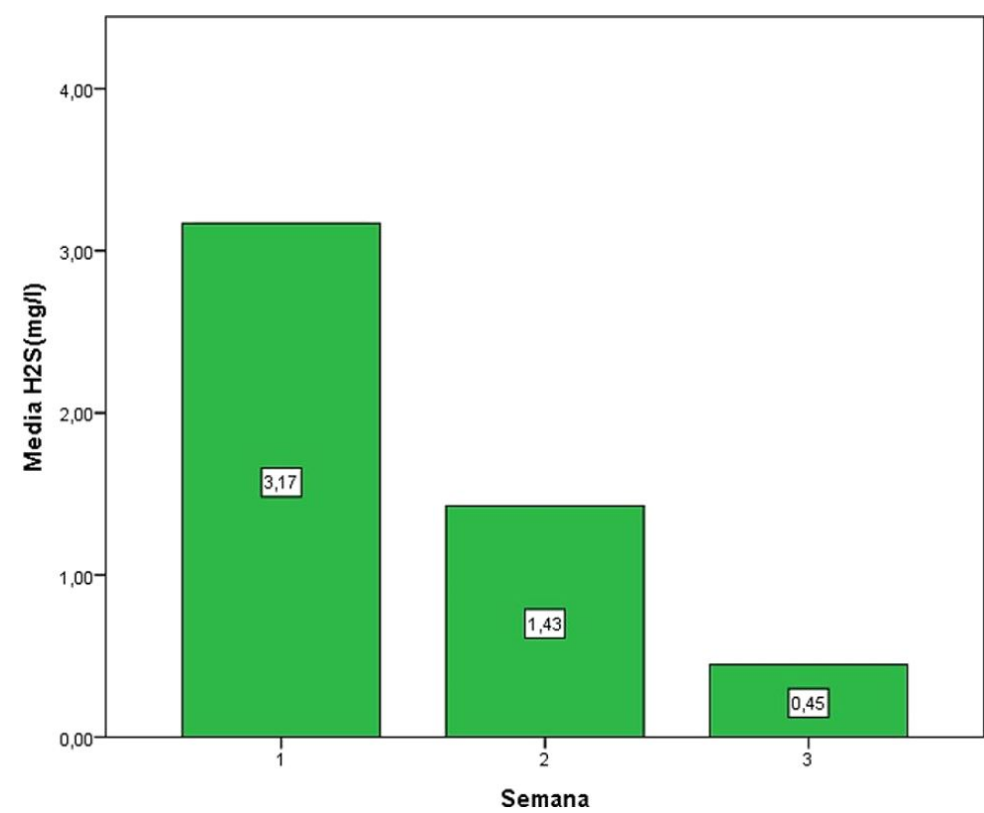

Figura 5. Media de la concentración de $\mathrm{H}_{2} \mathrm{~S}$ durante tres semanas de tratamiento con Microorganismos Eficientes. 
En la Figura 5 se puede observar la media de la concentración de $\mathrm{H}_{2} \mathrm{~S}$ analizado en aguas residuales de una industria procesadora de alimentos, en relación con las 3 semanas de tratamiento con microorganismos eficientes.

En la semana tres se presenta un valor de 0,45 $\mathrm{mg} / \mathrm{L}$ de concentración de $\mathrm{H}_{2} \mathrm{~S}$ ( $\mathrm{TRH}=12$ horas), considerándose significativamente bajo en comparación de la semana una y dos, con valores de 3,17 y $1,43 \mathrm{mg} / \mathrm{L}$ respectivamente, con un TRH de 6 horas en las dos semanas.

En las tres semanas de tratamiento existió reducción de la concentración de $\mathrm{H}_{2} \mathrm{~S}$, sin embargo, en la semana uno su reducción fue baja, debido a que los microorganismos fueron poblando y adaptándose al medio (Rehman, Farooqi y Ayub, 2009), siendo así que en la semana dos ya se observan cambios en la reducción de la concentración más altos, la semana tres fue la que mayor impacto tuvo, logrando reducir de una concentración inicial de $3,17 \mathrm{mg} / \mathrm{L}$ a una concentración final de $0,45 \mathrm{mg} / \mathrm{L}$, obteniéndose un porcentaje de remoción del 70,35\%; lo que nos indica que el biofiltro en el transcurso del tiempo alcanza mayor estabilidad y mejor crecimiento microbiano en la biopelícula (Welter et al., 2010) especialmente de bacterias sulfato-reductoras, bacterias fotosintéticas, ácido lácticas y levaduras, que aumentan su carga microbiana gracias a la materia orgánica nativa del lodo activado, siendo muy viable para que dicha materia orgánica sea retenida en las capas del biofiltro, evitando ser arrastrada al hacer pasar una nueva carga de agua residual por el biofiltro.

Los parámetros adicionales como $\mathrm{pH}$, conductividad y turbidez estuvieron directamente relacionados con la reducción de ácido sulfhídrico. En cuanto al $\mathrm{pH}$ tiene una relación directamente proporcional con el porcentaje de remoción de $\mathrm{H}_{2} \mathrm{~S}$; a medida que el porcentaje de remoción aumenta, el $\mathrm{pH}$ disminuye (Cardona Gómez y García Galindo, 2008). Al analizar la conductividad, presentó un aumento debido a que las actividades de los microorganismos eficientes contribuyen al incremento de nutrientes, iones en solución y transmisión de calor debido a la misma temperatura ambiental (Zamora et al., 2009). La reducción de la turbidez determinó que, a partir de los 15 días de haber empezado el tratamiento, existe mayor actividad de los microorganismos, ya que su adhesión estaba bien desarrollada, lo que es explica la reducción significativa de la concentración de $\mathrm{H}_{2} \mathrm{~S}$ en la semana tres del tratamiento (Peérez Zúñiga, 2016).

\section{Conclusiones}

Se determinó que los microorganismos contenidos en el producto EM (Microorganismos Eficientes), actúan en el lodo activado, aumentando la capacidad de formar la biopelícula, y el crecimiento de bacterias encargadas de la remoción del $\mathrm{H}_{2} \mathrm{~S}$ en las aguas residuales industriales.

Para asegurar el desarrollo y supervivencia de los microorganismos eficientes, sobre el lodo activado, se controló durante la investigación un pH ácido con rangos comprendidos entre 5,5-6,5, una temperatura cálida de $30-45^{\circ} \mathrm{C}$, humedad del $60 \%$.

Se analizó la concentración de ácido sulfhídrico de las aguas residuales industriales, antes y después del tratamiento con microorganismos eficientes, mostrando una concentración inicial de 3,68 $\mathrm{mg} / \mathrm{L}$ y una concentración final de $0,45 \mathrm{mg} / \mathrm{L}$, obteniendo mayor porcentaje de remoción en la tercera semana de tratamiento, con un valor del 70,35\%. El TRH utilizado en la tercera semana fue de 12 horas, por lo que se concluye que manejar mayores tiempos de retención, incrementa la capacidad de remoción en el tratamiento.

Se verificó que la utilización de microorganismos eficientes en aguas residuales, para la remoción de $\mathrm{H}_{2} \mathrm{~S}$, resulta rentable por sus tiempos cortos de actuación y bajos costos de aplicación, además que, al ser un tratamiento natural, no causa ningún daño en el medio ambiente.

En el transcurso de la investigación se reveló que el lodo utilizado como sustrato para los EM (Microorganismos Eficientes), redujo la cantidad de patógenos y aumentó el contenido de nutrientes presentes al inicio del tratamiento, concluyendo que el lodo residual por sus características puede ser reutilizado para abonar el suelo en la agricultura.

\section{Agradecimiento}

Se agradece a la Industria de Embutidos La Ibérica por los datos proporcionados para la realización de la investigación, por tanto se cita expresamente que no existen intereses comerciales con el producto EM·1, compuesto por bacterias fotosintéticas $\left(10^{3}\right)$, bacterias ácido lácticas $\left(10^{4}\right)$ y levaduras $\left(10^{3}\right)$, utilizados para la investigación ya que simplemente se usaron con un fin investigativo. 


\section{Referencias}

Arriagada Monreal, A. 2008. Tecnologías para el tratamiento de olores en aguas servidas. Tesis de grado. Universidad de Chile. [en línea] disponible en: https://goo.gl/epW2n7.

Bas, E. 2017. "Ingeniería de aguas residuales para combatir el ácido sulfhídrico." Aguasresiduales.info. [en línea] disponible en: https://goo.gl/ UsxFVS.

Cardona Gómez, J y L. A. García Galindo. 2008. Evaluación del efecto de los microorganismos eficaces (EM) sobre la calidad de un agua residual doméstica. Tesis de grado. Pontificia Universidad Javeriana. [en línea] disponible en: https://goo.gl/xBc2vt.

Coronel Pazmiño, N. P. 2015. Diseño e implementación a escala de un biofiltro Tohá en la ESPOCH para la depuración de aguas residuales domésticas procedentes de la comunidad Langos La Nube. Tesis de grado. Escuela Superior Politécnica de Chimborazo. [en línea] disponible en: https://goo.gl/ h4rXP8.

EEAITAJ. 2013. Micoorganismos Eficaces EM. Estación Experimental Agropecuaria para la Introducción de Tecnologías Apropiadas de Japón. [en línea] disponible en: https:/ /goo.gl/PXqAuy.

EMPROTEC. 2016. Guia de la Tecnología EM. EM Producción y Tecnología S. A. [en línea] disponible en: https://goo.gl/VZSo7H.

Fernández-Alba, A. R, P Letón García, R Rosal García, M Dorado Valiño, R Villar Fernández y J. M. Sanz García. 2006. Tratamientos avanzados de aguas residuales industriales. Informe de vigilancia tecnológica. Elecé Industria Gráfica. [en línea] disponible en: https:/ /goo.gl/epW2n7.

Galvis Toro, J y X. Rivera Guerrero. 2013. Caracterización fisicoquímica y microbiológica de los lodos presentes en la planta de tratamiento de aguas residuales industriales (ptari) de la empresa jugos hit de la ciudad de pereira. Tesis de Grado. Universidad Tecnológica de Pereira. [en línea] disponible en: https://goo.gl/GNTZUM.

García, Y, A Elías, N Albelo, F. R. Herrera, O Núñez y O. Dieppa. 2008. "Crecimiento de bacterias ácido lácticas y levaduras durante la fermentación líquida de excretas de pollos de ceba para la obtención de probióticos." Revista Cubana de
Ciencia Agrícola 42(2):195-197. [en línea] disponible en: https:/ /goo.gl/GWYdBt.

Higuera, O, J Arroyave y L. Flores. 2009. “Diseño de un biofiltro para reducir el índice de contaminación por cromo generado en las industrias del curtido de cueros." DYNA 76:107-119. [en línea] disponible en: https:/ /goo.gl/jbS5pk.

INEN. 2013. "Métodos Estandarizados para el Análisis de Aguas.".

Jusoh, Mohd Lokman Che, Latifah Abd Manaf y Puziah Abdul Latiff. 2013. "Composting of rice straw with effective microorganisms (EM) and its influence on compost quality." Iranian Journal of Environmental Health Science \& Engineering 10(1):17. [en línea] doi: https:/ /doi.org/10.1186/ 1735--2746--10--17.

Lázaro, L. A. 2014. Microbiotica, Nutrición Simbiótica y Microorganismos Regeneradores. Edicionesi. [en línea] disponible en: https://goo.gl/mxLqyo.

Madigan, M. T, J. M Martinko, J. P Thomas, B. C Rodríguez Fernández y M. Sánchez Pérez. 2004. Brock Biología de los Microorganismo. Pearson Educación. [en línea] disponible en: https://goo.gl/ LWxDiQ.

Moreno, J y K. Velarde. 2016. Aislamiento, caracterización y usos potenciales de microorganismos de tierra de montaña y subtrópico. Tesis de Grado. Escuela Politécnica del Chimborazo, Ecuador. [en línea] disponible en: https://goo.gl/SKRxXY.

Novoa, V, S Sunta, L Alomía y D. Baculima. 2008. Guía de prácticas ambientales unidades de producción agropecuaria. Dirección Metropolitana Ambiental. Quito - Ecuador. [en línea] disponible en: https://goo.gl/XjFGbk.

Organic Fruits. 2016. "Tecnología EM." Organic Fruits EIRL. [en línea] disponible en: https:/ /goo. $\mathrm{gl} / \mathrm{szN} 46 \mathrm{~s}$.

Pérez Zúñiga, M. E. 2016. Tratamiento de lodos residuales procedentes de plantas de tratamiento de aguas residuales mediante procesos electroquímicos para la disminución de metales pesados (PB). Tesis de Grado. Universidad Politécnica Salesiana, Ecuador. [en línea] disponible en: https://goo.gl/iQrJ2H.

Rehman, Z. U, I. H Farooqi y S. Ayub. 2009. “Performance of Biofilter for the Removal of Hydrogen 
Sulphide Odour." International Journal of Environmental Research 3(4):537-544. [en línea] disponible en: https://goo.gl/pPLfPA.

Salinas Carrillo, D y H. Huacollo Álvarez. 2011. Tratamiento de las aguas residuales del comité de regantes de Arunta. BIOEM Tacna, Tecnología EM para un ambiente sano y productivo. [en línea] disponible en: https://goo.gl/XjFGbk.

Szymanski, N y R. Patterson. 2003. Effective Microorganisms (EM) and Wastewater Systems. Future Directions for On-site Systems: Best Management Practice. [en línea] disponible en: https: //goo.gl/gx4kM9.

UTE. 2014. Métodos para la determinar el pH de una solución.
Varela Reyes, D. J. 2013. Evaluación de la viabilidad técnica y de costos de la aplicación de un proceso avanzado de oxidación fotocatalitico en el tratamiento de aguas residuales del sector textil de Bogotá. Master's thesis Universidad Nacional de Colombia. [en línea] disponible en: https: //goo.gl/9Y4Ksx.

Welter, A, J Romero, Y Grumelli y J. Sánchez. 2010. La biopelícula en los procesos RBC. Biblioteca virtual de desarrollo sostenible y salud ambiental. [en línea] disponible en: https://goo.gl/88QF8B.

Zamora, F. R, N. J Rodríguez Guevara, D. J Torres Rodríguez y H. J. Yendis Colina. 2009. “Uso de agua residual y contenido de materia orgánica y biomasa." Agricultura técnica en México 35(2):211-218. [en línea] disponible en: https:// goo.gl/oRQE7L. 